\title{
Rejection behaviour: a human homologue of the abnormal behaviour of Denny-Brown and Chambers' monkey with bilateral parietal ablation
}

\author{
ETSURO MORI, ATSUSHI YAMADORI \\ From the Neurology Service, Hyogo Brain and Heart Centre, Himeji, Japan
}

SUMMARY A unique behavioural syndrome in humans followed two separate strokes involving both $\stackrel{\mathbb{D}}{\circ}$ parietotemporal regions. The behavioural alterations resemble those of Denny-Brown and $\%$ Chambers' monkey with bilateral ablation of the parietal lobe which were characterised by strong $\vec{\circ}$ withdrawal or refusal to be touched on the limbs and head. In both humans and animals, touch on the $\overrightarrow{\vec{\omega}}$ limbs or head elicited exaggerated withdrawal movements and refusal to be touched on the lips and tongue resulted in difficulty in feeding. These behavioural alterations can be interpreted as loss of exploratory activities towards extrapersonal space, or more positively, as rejection of contact with the environment. This rejection behaviour in which tactile, visual, and/or gustatory exploration $\mathrm{N}$ tendencies are altered, is a counterpart of human frontal lobe syndrome and human Klüver-Bucy $\overrightarrow{.}$ syndrome.

In their series of studies on parietal lobe function, Denny-Brown and Chambers' noted that ablations of the parietal lobe in the rhesus monkey resulted in remarkable behavioural alterations. Following unilateral ablation of the parietal lobe, the animal developed distortion of behaviour characterised by avoiding reactions to stimuli in the opposite somatic and visual fields. After bilateral ablation the whole behaviour of the animal changed so that withdrawal was the response to any stimulus, including food which normally attracted the animal. The animal with bilateral ablation became nervous and restless with any form of stimulation, backing away from the stimulus. There was great sensitivity to contact with exaggerated withdrawal of head or limbs if these were touched (tactile avoiding reaction). Although the animal appeared not to see an object approaching him in the acute stage after operation, avoiding reaction thereafter was elicited by a visual stimulus (visual avoiding reaction). The avoiding reaction of the lips led to great difficulty in getting the animal to open its mouth and feed.

Avoiding responses due to parietal lobe damage are dynamically related to the grasping behaviours due to

Correspondence to: Etsuro Mori, Neurology Service, Hyogo Brain and Heart Centre at Himeji, 520, Saisho-Ko, Himeji, 670, Japan.

Received 15 November 1988 and in revised form 17 May 1989. Accepted 14 June 1989 frontal lobe damage. ${ }^{23}$ The pathological grasping behaviour has been interpreted as a feature of exage gerated tactile exploratory tendency inherent i $z$ parietal lobe function. ${ }^{13}$ This interpretation has been amply confirmed by monkey experiments. ${ }^{\prime}$ Recentt reported behavioural syndromes, such as compulsive manipulation of tools ${ }^{4}$ and utilisation behaviour, ${ }^{5}$ ca also be interpreted as a type of manual grasping behaviour in more elaborate form. In all these syndromes, the responsible lesions have been related to the frontal lobe.

On the other hand, bilateral temporal lobe damage has been known to produce the Klüver-Bucy syndrome. ${ }^{6}$ The syndrome includes psychic blindness, strong tendencies to examine all objects orally, an irresistible impulse to touch, loss of normal anger and fear responses and increased sexual activity. ${ }^{6}$ This syndrome, originally reported with experimental monkeys, was also confirmed in human patients with the same distribution of lesions. ${ }^{7}$ Part of the syndrome, such as hypermetamorphosis can be interpreted as a manifestation of exaggerated visual, tactile and oral exploring tendencies as a result of the loss of visual avoiding reaction. ${ }^{1}$

Thus clinical syndromes are known which are almost equivalent to the monkey syndromes in frontal and temporal lobe damage. However, we still do not know the human equivalent of the monkey parietal lobe syndrome produced by Denny-Brown and Chambers. Some researchers have expressed doubts that 


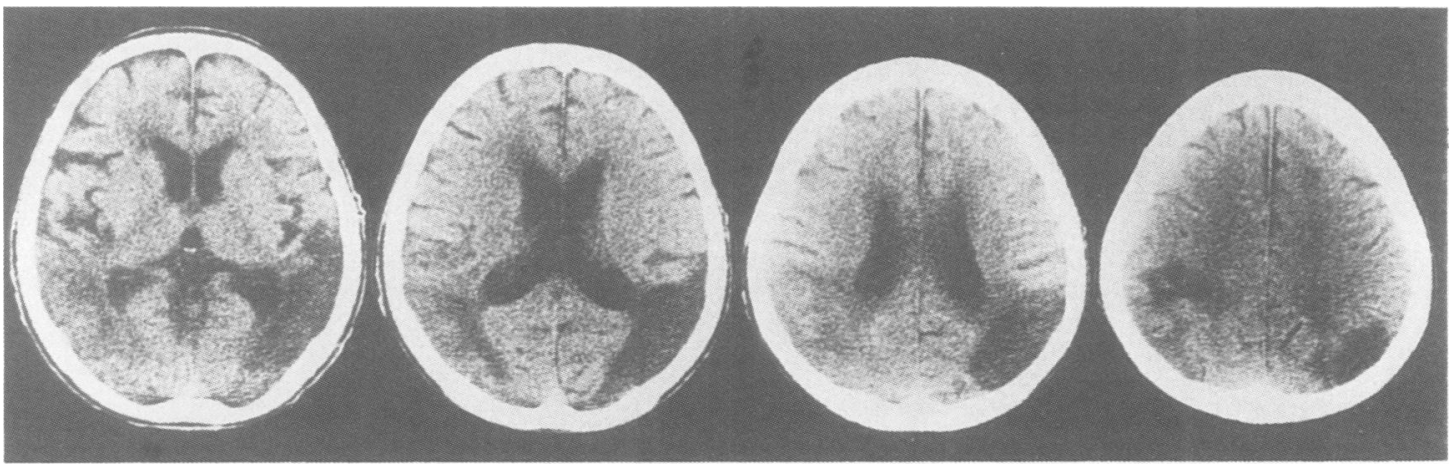

Fig 1 Contiguous CT sections of patient 1. Left appears to be on the left.

parietal lobe lesions could induce indifference or escape behaviour in response to tactile, visuo-tactile or visual stimuli. ${ }^{5}$ Although Denny-Brown ${ }^{3}$ maintained that repellent apraxia in man ranked with avoiding reaction in monkeys, it was rather different from the behaviour observed in experimental animals. The former is only a simple sensorimotor abnormality, ${ }^{89}$ that is, a manifestation of deafferentation, which appears following lesions in the posterior column of the spinal cord $^{1011}$ or thalamus, ${ }^{12}$ while the latter is highly integrated and elaborated and involves alteration of an individual's whole attitude to their environment. We describe four patients with strokes in the parietal lobe of both hemispheres whose changes in behaviour are comparable to that of Denny-Brown and Chambers' monkey with bilateral ablation of the parietal lobe. The changes in their behaviour were characterised by strong withdrawal from tactile stimuli. We propose to call this new human behavioural syndrome 'rejection behaviour'.

\section{Case reports}

Patient 1. A 72 year old right-handed man, with a history of atrial fibrillation, suddenly developed agitated delirium and was admitted to our hospital. His family had not noticed any change in his behaviour before this episode. On admission, he was agitated, resistant and restless. His speech was incoherent. He did not comprehend spoken language at all. Cranial nerves other than visual acuity, muscle strength, tendon reflexes and plantar responses were normal. Computed tomography (CT) of the brain showed two hypodense areas: one was a well-demarcated hypodensity in the right parietotemporooccipital region consisting of an old infarction in the territory of the inferior division of the right middle cerebral artery, and the other a faint hypodensity in the left parietotemporal region consistent with an acute infarction of the inferior division of the left middle cerebral artery (fig 1).

Within a few days, his initial agitated behaviour improved considerably. He was logorrhoeic, insomniac at night and incontinent but he was able to walk without help. However, he did not recognise any objects placed in front of him and did not make eye contact. His visual disturbance was interpreted as cortical blindness or severe visual inattention. He also appeared to be deaf but sometimes responded to loud noises. He was usually bad tempered.

A light touch consistently produced withdrawal movements in the head and limbs. There was no evidence that a light touch on the body produced a painful sensation. Because of these reactions, nursing was difficult and feeding impossible. The lips became tightly closed and he averted his face when food was placed on his lips. When a piece of food was pushed into his mouth, he would spit it out as if the taste

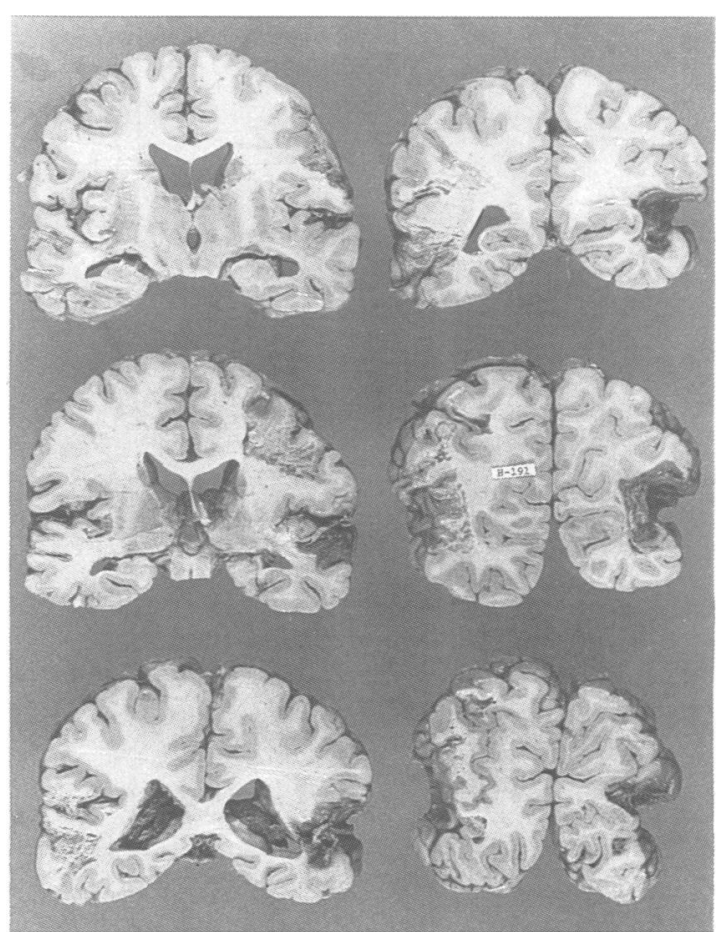

Fig 2 Coronal sections of the brain of patient 1. Old infarctions of the parietotemporooccipital region of both hemispheres. Relatively new infarctions also affecting the left rolandic area. 


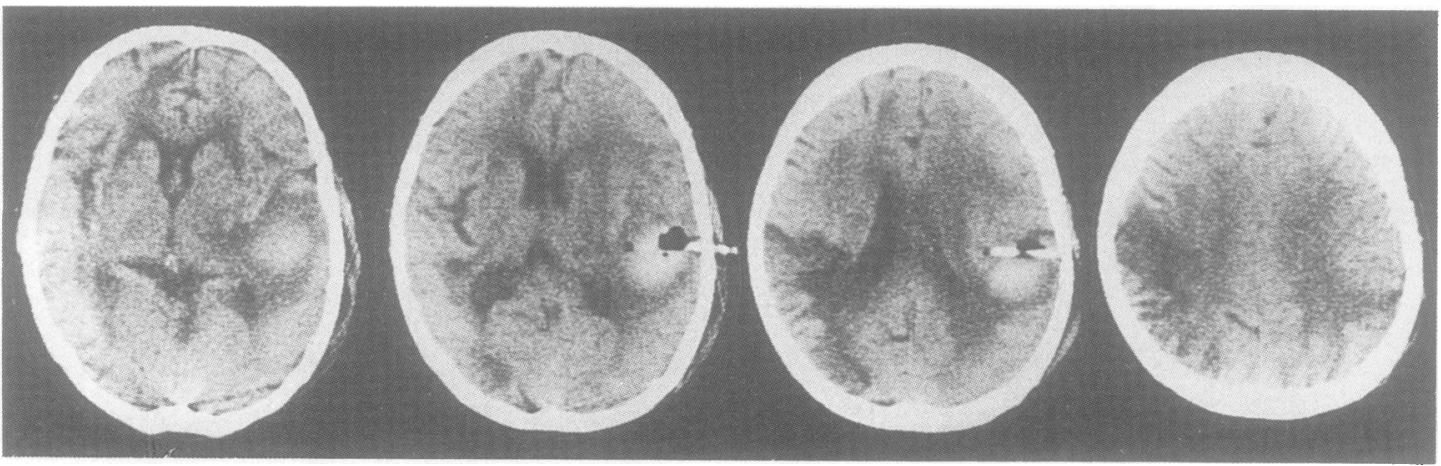

Fig 3 Contiguous CT sections of patient 2. Left appears to be on the left.

Patient 1
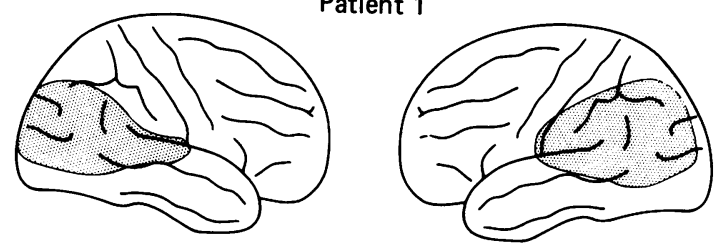

Patient 2
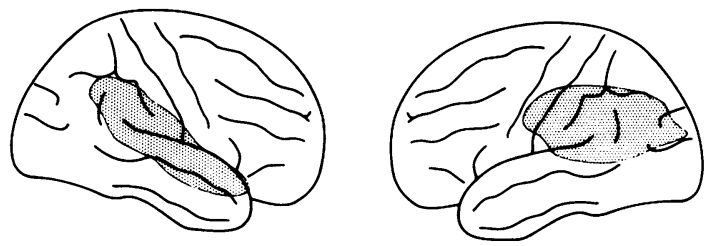

Patient 3
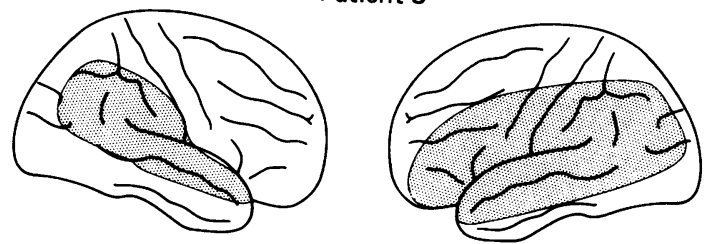

Patient 4
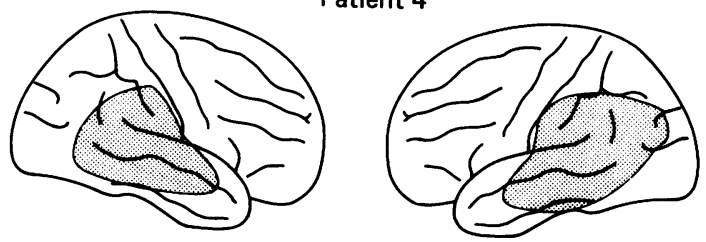

Fig 4 Schematic representation of lesion extent. Drawings based on material at necropsy in patient 1 and reconstructed from the CT using Mazzochi and Vignolo's method ${ }^{13}$ in patients 2, 3, and 4. These show a common lesion site in the inferior parietal gyrus and posterior part of the superior. temporal gyrus of both the hemispheres. was bitter. His gag reflex was within normal range and movements of the soft palate, lips and tongue were all normal, as was swallowing. It was unknown whether gustation was preserved. Intravenous nutrition and tube feeding were required for a month. This rejection behaviour lasted for approximately $\mathbf{4 0}$ days then gradually subsided and he was then able to feed himself. He was discharged home two months after the onset.

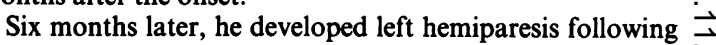
infarction in the right paracentral region and one year lateo $\vec{\overrightarrow{ }}$ he died of gastric cancer. Neuropathological examinatio $\overline{0}$. revealed: old ischaemic lesions with cystic change of the righ parietotemporooccipital region; an old infarction of the le fir parietotemporal region; an old infarction of the right Rolan dic area; a small, old infarction in the right cerebello $Z$ hemisphere (fig 2). Although moderate atherosclerosis was found in the basilar artery, occlusion was not apparent in the major cerebral arteries. The infarction of the right parietotemporal region affected the inferoposterior portion of the inferior parietal lobule, the posterior portion of the $\vec{\varphi}$ superior temporal gyrus and the anterolateral part of the occipital lobe. In the left hemisphere, the whole of the inferior parietal lobule, the posterior part of the parietal operculum, the posterior part of the superior temporal gyrus and the anterolateral part of the occipital lobe were involved. In both hemispheres, the Heschl gyrus was destroyed and the optic radiations were disconnected (fig 1).

Patient 2. A 71 year old right-handed hypertensive housewife was first admitted to this unit with a two week history of speech disturbance and altered behaviour. Examination revealed right homonymous hemianopia, right-sided mild hemisensory deficits, alexia with agraphia, constructional disability, ideomotor apraxia and mild right-sided visual neglect. CT showed a hypodensity in the left parietotemporal region consistent with an infarction in the territory of the inferior division of the left middle cerebral artery. She was discharged with a diagnosis of thrombotic stroke. Six months later, she suddenly became unconscious and was readmitted. She was stuporose. There were conjugate rightward ocular deviation, left hemiparesis, hyperreflexia on the left side and extensor plantar response on the left side. CT on the second admission showed a massive subcortical haematoma in the right parietal lobe as well as the previous left parietotemporal infarction (fig 3). Two weeks later, a burr hole was made and the haematoma was removed. 


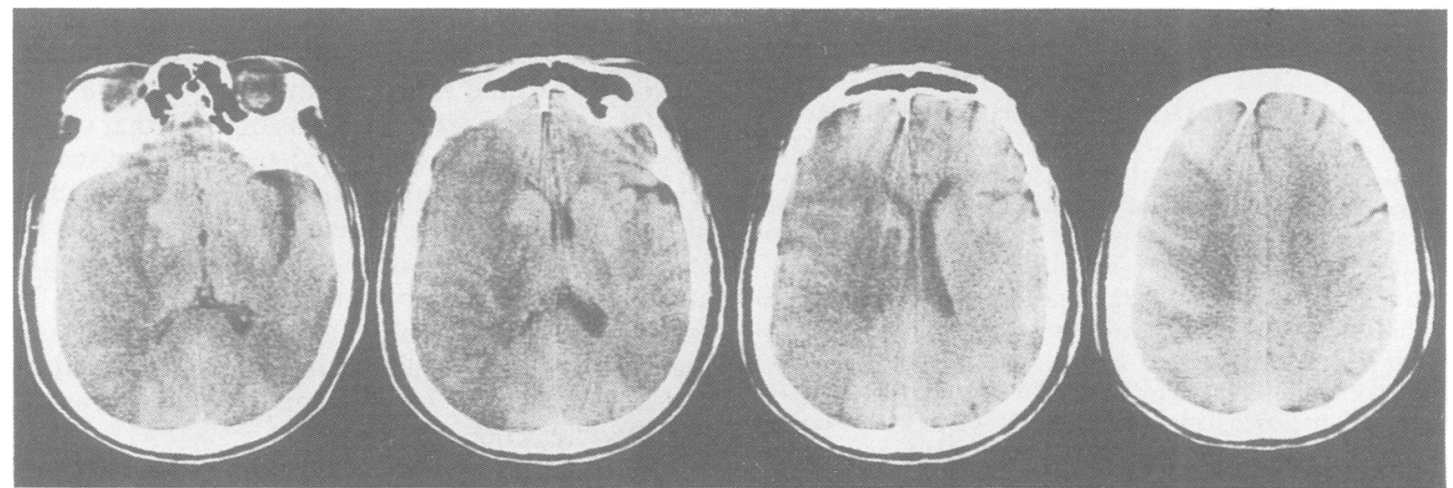

Fig 5 Contiguous CT sections of patient 3. Left appears to be on the left.

After remaining in a critical condition for a few weeks, she regained consciousness and muscle strength of the limbs of the left side. However, as her recovery progressed, behavioural abnormalities became evident. She was restless, incontinent, had insomnia, and was markedly logorrhoeic. Her speech was irrelevant and incoherent and she appeared to have both cortical blindness and deafness. Although she was frequently irritable, her facial expression appeared normal. Light touch consistently produced withdrawal movements of the limbs and head but did not seem to cause pain. When feeding was attempted, her lips remained firmly closed and she turned her face away. If the food was put into the mouth, she would push it out with the tongue. There was no apparent swallowing difficulty. Movements of the lips, soft palate and tongue were normal. It was not possible to test gustatory sensation. As a result it was necessary to feed her by nasogastric tube. This abnormal behaviour lasted for over a year.

An analysis of the CT scans (using the method described by Mazzochi and Vignolo ${ }^{13}$ ) showed that, in the left hemisphere damage had occurred to the whole of the inferior parietal lobule, the superior temporal gyrus and the parietal operculum. In the right hemisphere the areas affected were: the whole of the inferior parietal lobule, the inferior half of the paracentral gyri, and the posterior part of the superior temporal gyrus (fig 4).

Patient 3. A woman aged 72 years with a history of sick sinus syndrome was admitted to this unit because of the sudden onset of right hemiplegia. She had global aphasia, dense right hemiplegia, right hemisensory deficits, right homonymous hemianopia and conjugate leftward ocular deviation. Emergency angiography showed a distal occlusion of the left internal carotid artery. CT the day after showed a large perisylvian hypodensity consistent with an infarction of the territory supplied by the left middle cerebral artery. On the seventh day, the conjugate ocular deviation reversed its direction. Repeated CT showed another hypodensity in the right parietotemporal region indicating cerebral embolism in the region of the inferior division of the right middle cerebral artery (fig 5).

For a few weeks her condition was critical. She then became awake and her conjugate ocular deviation disappeared. She was incontinent and had insomnia and she restlessly moved her limbs. The right hemiplegia and global aphasia remained unchanged. She was mute and did not understand any verbal commands. She appeared to have cortical blindness and deafness. Her affect was flat and she withdrew her hand whenever it was touched. When touched on her left palm, she extended her fingers and wrist, to avoid the stimulus. She pushed food out with the tongue when it was forced into her mouth. She was fed through a nasogastric tube. Swallowing appeared normal. Gustation seemed preserved to some degree. When syrup was placed on her tongue, she appeared to appreciate the taste and when brine

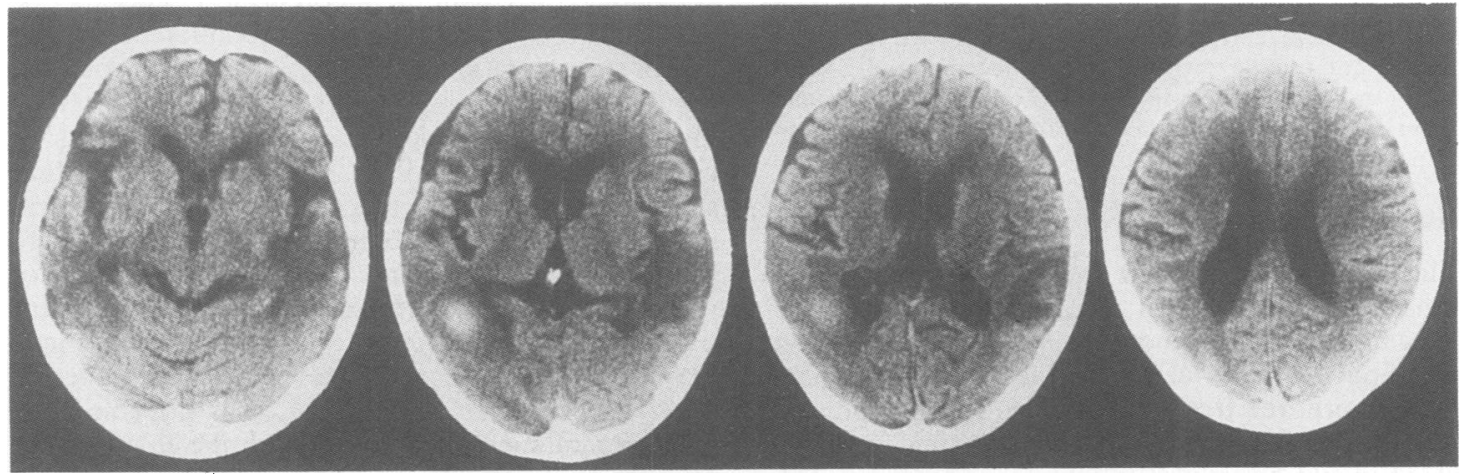

Fig 6 Contiguous CT sections of patient 4. Left appears to be on the left and right on the right. 
water was given she spat it out. Three months after the onset there was no improvement and she was transferred to a local hospital.

The CT scan analysis showed the lesion in the left hemisphere extended to the inferior frontal gyrus, paracentral gyri, inferior parietal lobule, and superior and middle temporal gyri; the lesion in the right hemisphere involved the parietal operculum, inferior parietal lobule and the whole of the superior temporal gyrus (fig 4).

Patient 4. A 73 year old right-handed woman was admitted to this unit with right-sided convulsions. Three months before admission she had had a cerebral infarction and Wernicke aphasia and had been admitted elsewhere for six weeks. CT disclosed haemorrhage in the left parietotemporal subcortical region as well as a hypodensity in the left parietotemporooccipital region consistent with the previous cerebral infarction. After the seizure was controlled there was right homonymous hemianopia and severe Wernicke aphasia with no comprehension of language. Angiography was performed on the tenth day and showed a dural arteriovenous malformation in the left posterior fossa. She then became restless, unable to sleep and incoherent. Her behaviour then changed considerably and she became irritable and unresponsive to bright light or loud noises. If touched on her limb, she would withdraw from the contact. Although she responded to light touch on her body with grimacing and crying, the appearance was quite different from that observed when truly painful stimuli were given. Therefore, it was unlikely that the light contact elicited a painful sensation. Touch on the face consistently produced withdrawal movement of her head. When her lips were touched, she would firmly close them. If food was put into her mouth, she would push it out with the tongue. Because food was refused intravenous nutrition and tube feeding were maintained. Our experience of the similar syndromes in those patients described above made us suspect a possibility of bilateral parietotemporal lesions. Indeed, the second CT on the 13th day showed a recurrence of a cerebral infarction involving the right parietotemporal region (fig 6). Withdrawal movements of the hands from tactile stimuli decreased by the end of the second week. However, avoiding movements of the face and lips persisted, and refusal to take food continued. She would only take a certain type of fruit and ice cream. The dural arteriovenous malformation was completely removed one month later. However, her behaviour pattern remained unchanged.

The analysis of the CT scans indicated that lesions in the left hemisphere extended to the inferior parietal lobule, superior and middle temporal gyri and occipital lobe, whilst lesions in the right hemisphere involved the parietal operculum, inferior parietal lobule and the whole of the superior temporal gyrus (fig 4).

\section{Discussion}

Bilateral parietotemporal lobe damage resulted in remarkable behavioural changes in four patients. Touching the limbs and head produced withdrawal behaviour. When touched on the hand, the patients quickly withdrew from the stimulus. These withdrawal movements were not a response to pain sensation which would occur with a thalamic syndrome. They refused food by closing the mouth tightly or by averting the face. If the food was placed in the mouth, the patients would expel it with the tongue. As there was no consistent reaction, it was unlikely that refusal to take food was due to an alteration of gustatory sense. This behavioural distortion must be a reflection of the alteration of the patient's attitude towards the environment. Withdrawal, or more positively, avoidance became a dominant response to contact. To the observer, these patients gave a strong impression that they were actively "rejecting" all external contact. Thus, we propose to call this peculiar symptom complex "rejection behaviour".

Such behaviour is equivalent to those responses observed in Denny-Brown and Chambers' experiments on animals with bilateral ablation of the parietal lobes. Operative removal of the parietal lobes in the monkey induced a lasting change in the behaviour of an animal characterised by similar withdrawal activity, associated with the facial expression, irregular exaggeration and repetition of withdrawal movements characteristic of anxiety. ${ }^{1}$ A light touch on a hand or foot led to exaggerated but delicate withdrawal from the stimulus (tactile avoiding). When tested with objects in the visual field the animal wouldo look away and push away with the hand (visua $\bar{\Phi}$ avoiding). Avoiding reaction of the lips led to great difficulty in feeding in the acute postoperative stage 0 Even after a long survival some difficulty remained in getting the animal to open its mouth.' Therefore, it is conceivable that the rejection behaviour is homos logous to the behaviour of the Denny-Brown and Chambers monkey. Associated emotional changes such as irritability, restlessness and insomnia, were also seen in the experimental animals.

There were, however, differences between the experimental animal and the patient with strokes. In the monkey, unilateral parietal damage produced avoiding reaction in the contralateral limbs, ${ }^{1}$ whilst in patients with strokes a unilateral parietotemporal lesion did not produce any changes in exploratory behaviour other than unilateral neglect. This may be either due to species difference or to differences in the size of the lesion (in our patients the superior parietal lobule was always spared). Although detailed assessments were impossible because of gross deficits of higher cortical function, there was no doubt that severe visual and auditory disturbances of central origin, that is, cortical blindness and deafness, were present in all our patients. These features were not recorded in the experimental animal. Concurrent damage of visual, auditory and linguistic functions may be a necessary condition for the appearance of rejection behaviour in humans. It is possible that, when communicative channels with the environment other than the tactile modality are open, this tactile 
avoiding tendency may be easily inhibited by some kind of compensatory mechanism. A relationship between gustatory alteration and rejection of food remains uncertain. Gustatory alteration was not described in the experiments of Denny-Brown and Chambers, but it is quite possible that parietal lesions may change gustation. The gustatory sensation is thought to reside in the anterior Sylvian cortex ${ }^{14}$ or parietal operculum. ${ }^{1516}$

In the present series, common lesions always included the inferior parietal lobule and posterior part of the superior temporal gyrus suggesting the importance of these lesions in producing rejection behaviour (fig 4). The experiments of Denny-Brown and Chambers ${ }^{1}$ indicated a relatively greater release of visual avoiding reaction by ablation of the posterior parietal lobe (including the posterior part of the superior temporal sulcus), of tactile and nociceptive reactions by anterolateral ablation (namely, the postcentral gyrus and parietal operculum) and of all types of avoiding reaction by ablation of the intraparietal area (area 7). The areas in the monkey brain which can be considered homologous to the inferior parietal lobule of the human brain are the inferior parietal lobule and the depths of the superior temporal sulcus. ${ }^{17}$ In addition, the equivalent area of monkey intraparietal area is considerably larger in the human brain. ${ }^{18}$ Brodmann $^{19}$ pointed out that his monkey area 7 should be thought of as an undifferentiated area comparable to his human areas 7,39 and 40 . Therefore, the extent of lesions indicated in this study is comparable to ablation of the posterior parietal and intraparietal regions in the monkey.

Based on animal models with cortical ablations, Denny-Brown and Chambers' postulated that, in the monkey, the parietal lobes provide for stereotactic exploratory behaviour and orientation in space, including the optic righting reflex and that the cingulate gyrus and supplementary motor area serve the tactile avoiding responses and the temporal lobe serves the visual avoiding responses. Their experiments suggest that the whole behaviour reaction to exteroceptive events is determined by a balanced cortical response to the environment. Disturbance of this equilibrium by removal of one or other of the areas concerned with positive and negative types of response results in transcortical release and a relative preponderance of its natural competitor. The frontal, parietal and temporal lobe syndromes are determined, in part, by the absence of specific behavioural responses mediated by the area concerned and partly by the release of subcortical mechanism of the lost function and by transcortical release. This hypothesis fits with rejection behaviour, as well as the human frontal lobe and Klüver-Bucy syndromes. The parietal lobe has a critical role in extrapersonal spatial (tactile, visual, and visuospatial) processing. ${ }^{23}$ Thus rejection behaviour is an extreme form of the parietal lobe syndrome and can be interpreted as a total loss of exploration to the environment, or more positively, as a refusal of contact with the environment. It is a counterpart of the frontal lobe syndrome and the Klüver-Bucy syndrome in which tactile and visual exploration are hyperactive. Some of the frontal lobe syndromes including oral tendency, sucking response, bilateral instinctive grasp reaction, ${ }^{2}$ compulsive manipulation of tools, ${ }^{4}$ utilisation behaviour ${ }^{5}$ and some of the main fragments of the Klüver-Bucy syndrome including hypermetamorphosis, hyperorality and hyperbulimia are in sharp contrast with the main symptoms of rejection behaviour. In addition, emotional characteristics of irritability and temper observed in rejection behaviour are in contrast with the placidity and apathy of those with frontal lobe or Klüver-Bucy syndromes.

The behavioural syndrome we describe could be called the "Denny-Brown Chambers syndrome." This behaviour is occasionally observed in severe neurological diseases. More attention should be given to the role of the parietal lobe in human behaviour. From a clinical point of view, the main problem in rejection behaviour is care and nursing. It is important, therefore, for those who care for patients with diseases that predominantly affect the parietal lobe to recognise this rejection behaviour.

\section{References}

1 Denny-Brown D, Chambers RA. The parietal lobe and behaviour. Res Publ Assoc Res Nerv Ment Dis 1958; 36:35-117.

2 Seyffarth H, Denny-Brown D. The grasp reflex and instinctive grasp reaction. Brain 1948;71:109-83.

3 Denny-Brown D. The nature of apraxia. J Nerv Ment Dis 1950;126:9-32.

4 Mori E, Yamadori A. Compulsive manipulation of tools and pathological grasp phenomenon. Clin Neurol 1982;22:329-35.

5 Lhermitte F. Utilization behaviour and its relation to lesions of the frontal lobes. Brain 1983;106:237-55.

6 Klüver H, Bucy PC. Preliminary analysis of functions of the temporal lobes in monkeys. Arch Neurol Psychiatr 1939;42:979-1000.

7 Lilly R, Cummings JL, Benson F, Frakel M. The human Klüver-Bucy syndrome. Neurology 1983;33:1141-5.

8 Dehen H, Willer JC, Cambier J. Cutaneous reflexes in the avoidance reaction. Eur Neurol 1981;20:416-20.

9 Laplane D, Meininger V, Bancaud J, Talairach J, Broglin D. Contribution à l'étude anatomoclinique des phénomènes d'évitement. Rev Neurol (Paris) 1979; 135:775-87.

10 Cambier J, Dehen H. Imitation synkinesia and sensory control of movement. Neurology 1977;27:646-9.

11 Gilman S, Denny-Brown D. Disorders of movement and 
behaviour following dorsal column lesions. Brain 1966; 89:397-417.

12 Denny-Brown D. The cerebral control of movement. Liverpool: Liverpool University Press, 1966.

13 Mazzochi F, Vignolo LA. Computer assisted tomography in neuropsychological research: a simple procedure for lesion mapping. Cortex 1976;14:136-44.

14 Bagshaw MH, Pribram KH. Cortical organization in gustation (Macaca mulatta). J Neurophysiol 1953;16: 499-508.

15 Bornstein WJ. Cortical representation of taste in man and monkey. I. Functional and anatomical relations of taste, olfaction, and somatic sensibility. Yale $J$ Biol
Med 1940;12:719-36.

16 Bornstein WJ. Cortical representation of taste in man and monkey. II. The localization of the cortical taste area in man and a method of measuring impairment of taste in man. Yale J Biol Med 1940;13:133-56.

17 Mesulam M-M. A cortical network for directed attention and unilateral neglect. Ann Neurol 1981;10:309-25.

18 Jones EG, Powell TPS. An anatomical study of converging sensory pathways within the cerebral cortex of the monkey. Brain 1970;93:793-820.

19 Brodmann K. Vergleichende Lokalizationslehre der Grosshirnrinde in ihren Prinzipien dargestellt auf Grund des Zellenbaues. Leipzig: J A Barth, 1925. 